\title{
Selective Analysis of Molecular States by Functionalized Scanning Tunneling Microscopy Tips
}

\author{
Z. T. Deng, ${ }^{1}$ H. Lin, ${ }^{2}$ W. Ji, ${ }^{1}$ L. Gao, ${ }^{1}$ X. Lin, ${ }^{1}$ Z. H. Cheng, ${ }^{1}$ X. B. He,,${ }^{1}$ J. L. Lu, ${ }^{1}$ D. X. Shi, ${ }^{1}$ W. A. Hofer, ${ }^{2}$ and H.-J. Gao ${ }^{1} * *$ \\ ${ }^{1}$ Beijing National Laboratory for Condensed Matter Physics, Institute of Physics, Chinese Academy of Sciences, \\ P.O. Box 603, Beijing 100080, China \\ ${ }^{2}$ Surface Science Research Centre, The University of Liverpool, Liverpool L69 3BX, United Kingdom \\ (Received 19 January 2006; revised manuscript received 24 March 2006; published 19 April 2006)
}

\begin{abstract}
Selective analysis of molecular states in scanning tunneling microscopy (STM) has so far been achieved in a few cases by tuning the bias range of the STM in high-resolution measurements. Correspondingly, perylene adsorbed in a close-packed monolayer on $\mathrm{Ag}(110)$ is imaged mainly through the $\pi$ states of the molecule. By contrast, functionalizing the STM tip with a perylene molecule leads to a mismatch between the energy levels of the STM tip and the molecule adsorbates and, instead, images only the metal states of the underlying silver surface. The observation opens a route for better energy selectivity in electron transport measurements through organic interfaces.
\end{abstract}

Organic molecules on metal surfaces have been extensively investigated due to their importance for nanotechnology, electrochemistry, biochemistry, and heterogeneous catalysis $[1,2]$. Since 1982 , many investigations in this area are performed by scanning tunneling microscopy (STM), which has become one of the most ubiquitous tools in surface science. The continuous refinement of analyzing techniques in STM has made it possible to analyze even atomic-scale electronic properties of adsorbate-substrate systems. A number of investigations have recently focused on selective measurements of individual molecular states or metal states (see, e.g., [3,4]). These experiments are of great interest for molecular electronics and nanoscale device fabrication [5-8]. However, selectively imaging molecular or metal states in STM measurements is usually prevented by the complex coupling of electronic states of metal surfaces, organic adsorbates, and metal tips. To our knowledge, a selective analysis of molecular states on metal surfaces has so far been achieved only in a very few cases by tuning the bias range of the STM. By contrast, functionalizing metal electrodes with organic polymers exhibits a high selectivity, e.g., in electrochemistry $[9,10]$. This method also seems suitable to produce functional STM tips, which are sensitive to molecular or substrate electronic states.

In this Letter, we demonstrate by low-temperature STM analysis and density functional theory (DFT) calculations that molecular $\pi$ states of perylene adsorbed on $\operatorname{Ag}(110)$ can be directly imaged by a clean tungsten tip. Functionalizing the tip by attaching a perylene molecule to its apex modifies the tip electronic structure. Subsequent changes of the scanning conditions (i.e., bias voltage and feedback current) allow selecting transitions from molecular states of perylene adsorbates or states of the Ag surface. This procedure opens a new route to discriminate between electronic states of organic adsorbates and states of the metal surface.

Our experiments are performed in a combined ultrahighvacuum-molecular-beam-epitaxy and low-temperature- scanning-tunneling-microscopy system. The base pressure in the experiments is below $3 \times 10^{-10} \mathrm{mbar}$. Prior to the adsorption experiment, the silver single crystal is cleaned by repeated cycles of $\mathrm{Ar}^{+}$sputtering $(1 \mathrm{keV}, 20 \mathrm{~min})$ and subsequent annealing at $700 \mathrm{~K}(20 \mathrm{~min})$. The bare silver surface is imaged before dosing to confirm its cleanliness. Perylene is thermally evaporated at $395 \mathrm{~K}$ onto the $\operatorname{Ag}(110)$ surface.

Previous low energy electron diffraction (LEED) experiments showed that, on an $\operatorname{Ag}(110)$ surface, the local adsorption configuration of perylene molecule does not depend much on the coverage $[11,12]$. The coverage of one molecular monolayer (ML) corresponds to about $5.0 \times$ $10^{13}$ molecules per $\mathrm{cm}^{2}$. To isolate the interaction between molecules and the surface from intramolecular interactions, we chose the coverage of $0.6 \mathrm{ML}$ to search for the most optimized adsorption configuration. For technical details of the calculations, see Ref. [13]. In total, twenty possible structures varying in orientations and adsorption sites have been analyzed. We find that the geometry of the molecule leads to an energy penalty of about $0.3 \mathrm{eV}$, if the central benzene ring is adsorbed on top of the silver rows, and that the molecule is preferably adsorbed with its longitudinal axis parallel to these rows. The physical reason for this behavior is the overlap between the $\pi$ states of the molecule and the electronic states of the surface. In the ground state adsorption geometry, this overlap is maximized, leading to the maximum binding energy. Here we present only the most stable configuration. As shown in Fig. 1(a), the center of perylene molecule is positioned above an fcc hollow site of the $\operatorname{Ag}(110)$ surface. The molecule adopts an orientation in which its long axis aligns parallel to the [110] direction of the $\operatorname{Ag}(110)$ surface. The adsorption energy per molecule in our calculation is $-0.53 \mathrm{eV}$, which is consistent with thermal-desorption experiments [11]. The weak adsorbate-surface interaction is due to the mismatch of molecular energy levels near the Fermi energy and energy levels of the Ag substrate [17]. Comparing the partial charge density of a perylene adsor- 

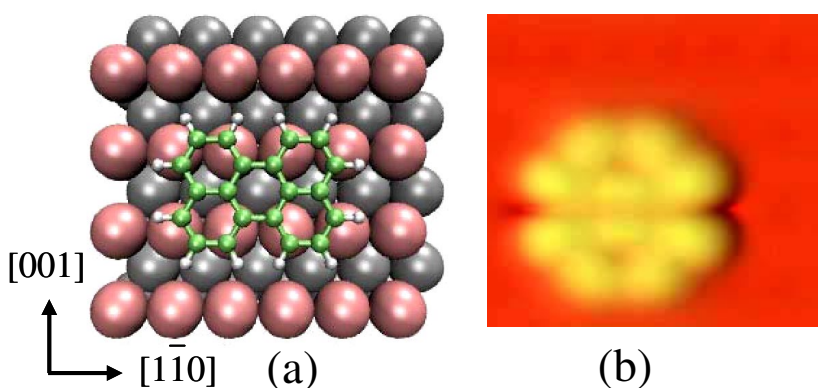

(b)
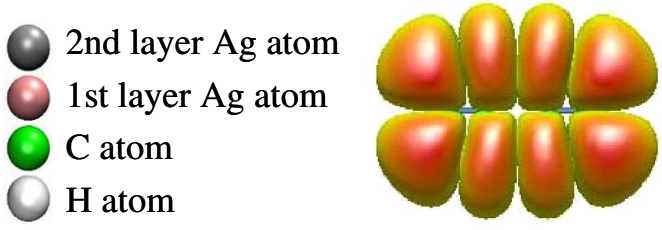

(c)

FIG. 1 (color online). (a) Ground state adsorption configuration of perylene molecule on the Ag (110) surface. (b) Charge density of perylene molecule deposited on the Ag (110) surface. (c) Charge density of an isolated perylene molecule in vacuum (from -0.7 to $+0.7 \mathrm{eV}$ relative to the Fermi level).

bate on the $\operatorname{Ag}(110)$ surface [see Fig. 1(b)] and an isolated perylene molecule in the vacuum [see Fig. 1(c)], it can be seen that the molecular states of perylene remain virtually unchanged rather than hybridize with the electronic states of the Ag surface.

Figure 2(a) shows a high-resolution STM image of a perylene monolayer on the $\operatorname{Ag}(110)$ surface. Each perylene molecule is imaged as four bright lobes with an apparent height of $1.5 \AA$. STM simulations indicate that this image is obtained with a clean tungsten tip [see Fig. 2(c)]. The twist of the molecule in the experimental STM image is probably due to the lateral intermolecular interactions of perylene adsorbates. In a close-packed configuration, the interaction between the hydrogen atoms at separate molecules will become the main component in the energetic stability of the molecular layer. A twist of the carbonhydrogen bonds in this case will distort the $\pi$ bonds of the molecule. Since the molecule is imaged mainly via these states, the molecule itself may appear twisted.

Comparing Fig. 2(c) with Fig. 1(b), it can be seen that the simulated STM image with a clean tungsten tip is roughly equivalent to the local charge density, i.e., the $\pi$ states, of perylene molecules on $\operatorname{Ag}(110)$ surface.

At dilute coverage, there is no long-range ordered structure of perylene adsorbates observed by LEED on the $\operatorname{Ag}(110)$ surface [11]. Since the disordered perylene molecules are now far away from each other, a tungsten tip can easily be functionalized by picking up a single perylene molecule from the silver substrate. Since tungsten is less noble than silver, the adsorption energy of perylene on tungsten is substantially higher. The actual adsorption

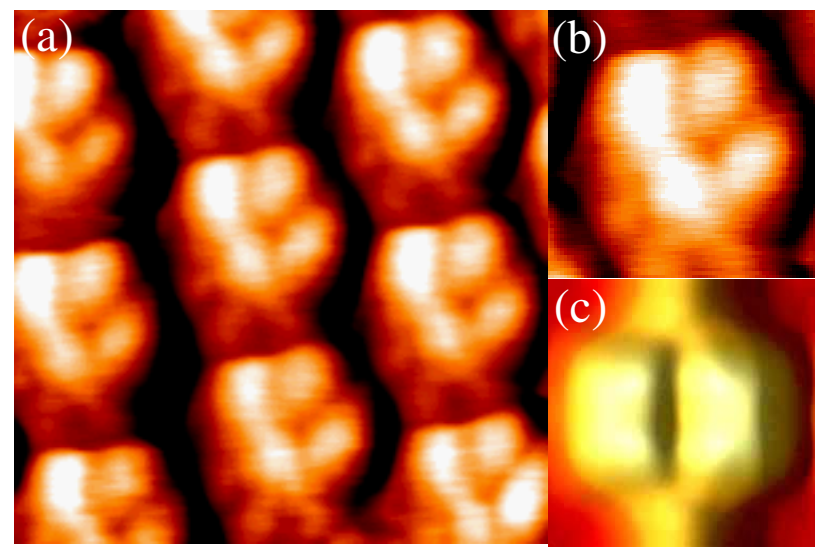

FIG. 2 (color online). A monolayer of perylene molecules adsorbed on Ag (110) surface. (a) A high-resolution STM image, $3 \mathrm{~nm} \times 3 \mathrm{~nm}$. (b) An enlarged image of one perylene molecule of the perylene monolayer in (a), $1.2 \mathrm{~nm} \times 1.2 \mathrm{~nm}$. (c) Simulated STM image with a clean tungsten tip. Parameters: $V=$ $+1.8 \mathrm{~V}, I=90 \mathrm{pA}$.

energy of perylene on a tungsten tip will be less than $-8 \mathrm{eV}$, calculated from the adsorption on a flat tungsten (100) surface, due to tip asperities. If only one benzene ring adsorbs to the tip apex, the adsorption energy will be in the range of $-1 \mathrm{eV} \mathrm{[18]} \mathrm{or} \mathrm{double} \mathrm{the} \mathrm{value} \mathrm{for} \mathrm{the} \mathrm{adsorption}$ on $\operatorname{Ag}(110)$. Therefore, the tip can be functionalized by scanning the surface at low coverage with a clean tungsten tip and at sufficiently high currents. At the beginning of the STM scanning, the molecules are imaged as protrusions on the surface. After we modify the tip by applying voltage pulses from the STM tip, the STM images change to a different mode. Thus, we conclude that the ensuing STM images are obtained with a different tip. The only option in this case is a tip functionalized by a perylene molecule. Changing the bias voltage and current, this functionalized tip can be used to discriminate the energy levels of perylene adsorbates. As shown in Fig. 3(a) (bias voltage $-0.8 \mathrm{~V}$, current $27.5 \mathrm{pA}$ ), part of the $\mathrm{Ag}$ surface now appears as a protrusion, and the perylene molecules seem to be embedded in the substrate. Figure 3(d) gives a highresolution detail of Fig. 3(a). Figure 3(g) is our STM simulation with a functionalized tungsten tip. Varying the bias voltage to $-0.67 \mathrm{~V}$ and changing the current to 161.5 pA [see Fig. 3(b)], the whole Ag surface appears, and the image of $\mathrm{Ag}$ rows in [110] direction is obtained. If the bias voltage is further increased to $-1.5 \mathrm{~V}$, a current of $149 \mathrm{pA}$ yields a high resolution of the Ag surface while the perylene adsorbates are seen as depressions [see Fig. 3(c)]. Simulations under identical tunneling conditions are shown in Figs. 3(h) and 3(i). All features are reproduced by STM simulations with a perylene-modified tungsten tip. While the central part of the molecule is still visible in Fig. 3(h), it has vanished in Fig. 3(i). The reason for this behavior is the onset of the $d$ band on the silver surface, which reaches its maximum density of states around 

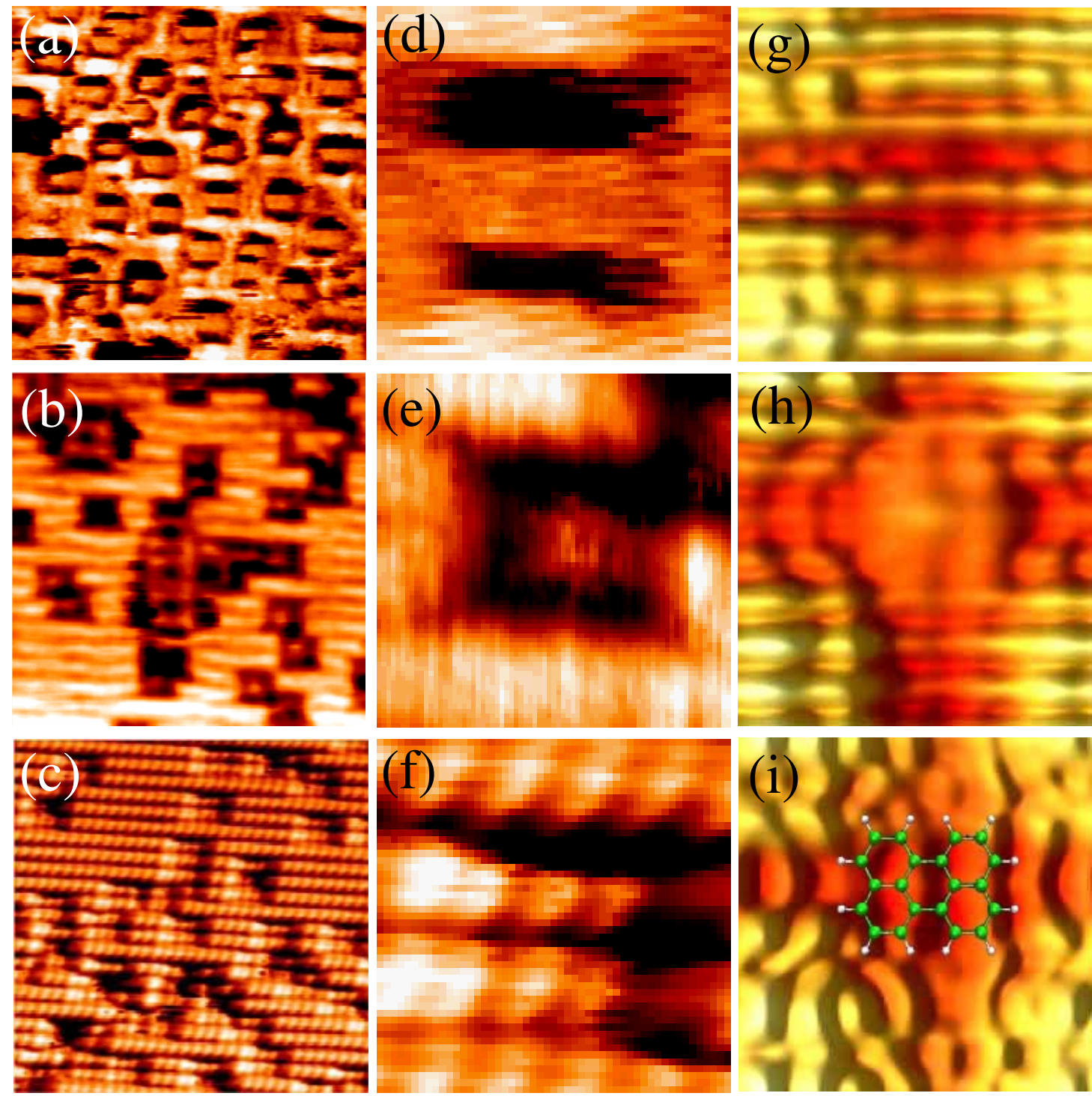

FIG. 3 (color online). (a)-(c) STM images of perylene molecules adsorbed on Ag (110). (d)-(f) High-resolution details of a single feature in (a)-(c). (g)-(i) STM simulations of (d)-(f) with a tungsten tip functionalized by perylene. The position of the perylene molecule on the surface is indicated (i). Parameters: (a) $10 \mathrm{~nm} \times 10 \mathrm{~nm}, 0.5 \mathrm{ML}, V=-0.8 \mathrm{~V}, I=27.5 \mathrm{pA}$; (b) $7.9 \mathrm{~nm} \times 7.9 \mathrm{~nm}$, $0.6 \mathrm{ML}, V=-0.67 \mathrm{~V}, I=161.5 \mathrm{pA}$; (c) $10 \mathrm{~nm} \times 10 \mathrm{~nm}, 0.3 \mathrm{ML}, V=-1.5 \mathrm{~V}, I=149 \mathrm{pA}$.

$-1.5 \mathrm{eV}$. At a bias value of $-1.5 \mathrm{~V}$, the protrusion in the middle of the molecule disappears, and the STM images reveal only the atomic positions of the silver surface. The images clearly demonstrate the induced functionality of the tip due to the adsorbed molecule. While the states of the molecule at the surface become invisible due to the increased energy difference to the states of the tip, the continuous $d$ band of $\mathrm{Ag}(110)$ gradually provides the majority of tunneling electrons. Correspondingly, we also note that varying the adsorption geometry of perylene on the tungsten tip, e.g., by rotating the molecule with respect to the vertical axis or changing the adsorption site, does not affect the results of the STM simulations. This indicates that most of the tunneling current is transported from the attached perylene molecule, while the influence of the tungsten tip configuration is rather small.

To understand the nature of this intriguing effect, we performed a partial charge density calculation for a perylene molecule on a W(100) film [see Fig. 4(a)]. As shown in Fig. 4(b), the $\pi$ orbitals of the perylene molecule merge completely with the charge density of the metal surface, which therefore greatly changes the charge density of the clean tungsten tip. The functionality of this tip, i.e., the discrimination of perylene states, is therefore due to the mismatch of energy levels of perylene molecules on the $\mathrm{Ag}$ surface and on the modified STM tip.

In summary, we have shown that the electronic states of perylene adsorbates and $\mathrm{Ag}$ substrates can easily be disen- 


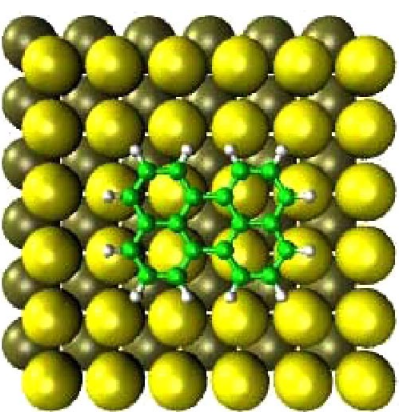

(a)

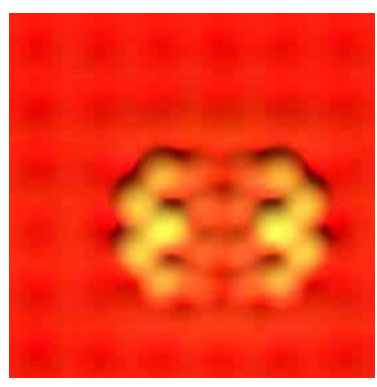

(b)
FIG. 4 (color online). (a) Perylene molecule attached to a $\mathrm{W}(100)$ surface. (b) Partial charge density of the perylenemodified tungsten surface (from -0.7 to $+0.7 \mathrm{eV}$ relative to the Fermi level).

tangled by using modified STM tips. The $\pi$ states of the perylene adsorbate can be imaged with a clean tungsten tip. Functionalizing the tip by attaching a perylene molecule on its apex, high-resolution images of $\mathrm{Ag}(110)$ surface states are obtained. This indicates that functionalizing STM tips with organic molecules can open up a new route for energy selection of transport properties through organic interfaces.

This work is funded by the Natural Science Foundation of China, the Chinese National Projects No. 863 and No. 973, and the Supercomputing Center, CNIC, CAS. H. L. acknowledges support from the ORS. W. A. H. thanks the Royal Society for financial support. The authors are grateful to C. Joachim for helpful discussions.

*Electronic address: hjgao@aphy.iphy.ac.cn

[1] J. K. Gimzewski and C. Joachim, Science 283, 1683 (1999).

[2] F. Rosei, M. Schunack, Y. Naitoh, P. Jiang, A. Gourdon, E. Laegsgaard, I. Stensgaard, C. Joachim, and F. Besenbacher, Prog. Surf. Sci. 71, 95 (2003).

[3] S. G. Lemay et al., Nature (London) 412, 617 (2001).

[4] G. V. Nazin, X. H. Qiu, and W. Ho, Science 302, 77 (2003).

[5] C. Joachim, J. K. Gimzewski, and A. Aviram, Nature (London) 408, 541 (2000).

[6] E. G. Emberly and G. Kirczenow, Phys. Rev. Lett. 91, 188301 (2003).
[7] J. Kuntze, R. Berndt, P. Jiang, H. Tang, A. Gourdon, and C. Joachim, Phys. Rev. B 65, 233405 (2002).

[8] J. Repp, G. Meyer, S. M. Stojković, A. Gourdon, and C. Joachim, Phys. Rev. Lett. 94, 026803 (2005).

[9] Handbook of Conducting Polymers, edited by T. A. Skotheim, R.L. Elsenbaumer, and J.R. Reynolds (Marcel Dekker, New York, 1998), 2nd ed.

[10] J. F. Rubinson and H.B. Mark, Jr., Conductive Polymers and Polymer Electrolytes: From Biology to Photovoltaics (American Chemical Society, Washington, DC, 2002).

[11] C. Seidel, R. Ellerbrake, L. Gross, and H. Fuchs, Phys. Rev. B 64, 195418 (2001).

[12] H. Han, M. Hongying, C. Qiao, Y. Xinzheng, Q. Huiqin, Z. Jianhua, L. Haiyang, H. Pimo, and B. Shining, Physica (Amsterdam) 352B, 36 (2004).

[13] Ab initio DFT calculations are carried out with the Vienna $A b$ Initio Simulation Package (VASP) [14]; the $\operatorname{Ag}(110)$ supercells are modeled by a four-layer Ag film with perylene molecules adsorbed on only one side of the surface. This geometry is justified in view of the very low adsorption energies and, consequently, low hybridization of electronic states, which does not give rise to surface dipoles. A vacuum of $17 \AA$ is used to separate the mirror images in the $z$ direction. The ion-electron interactions are described by ultrasoft pseudopotentials. The exchange-correlation potentials are calculated with the generalized gradient approximation according to Perdew, Burke, and Wang [15]. The energy cutoff for plane waves is $400 \mathrm{eV}$. The number of $k$ points is varied until the adsorption energies of perylene are converged. The same method is also used to calculate the electronic properties of STM tips. All STM simulations presented here are performed with a scattering method developed to first order in the Green's functions [16].

[14] G. Kresse and J. Hafner, Phys. Rev. B 47, R558 (1993); G. Kresse and J. Furthmüller, Phys. Rev. B 54, 11169 (1996).

[15] J.P. Perdew, J. A. Chevary, S. H. Vosko, K. A. Jackson, M. R. Pederson, D. J. Singh, and C. Fiolhais, Phys. Rev. B 46, 6671 (1992); J.P. Perdew, K. Burke, and Y. Wang, Phys. Rev. B 54, 16533 (1996).

[16] K. Palotás and W. A. Hofer, J. Phys. Condens. Matter 17, 2705 (2005).

[17] The weak interaction may also be due to the geometry mismatch of adsorbate and substrate. In the present case, perylene on $\operatorname{Ag}(110)$ and $\mathrm{W}(100)$, the difference of interaction strength is mainly due to the energy level mismatch.

[18] F. Mittendorfer and J. Hafner, Surf. Sci. 472, 133 (2001). 